\title{
柠檬酸浓度对硼酸盐玻璃骨水泥性能的影响
}

\author{
李海滨 ${ }^{1}$, 王德平 ${ }^{1,2}$, 吴莹莹 ${ }^{1}$, 姚爱华 ${ }^{1}$, 叶 松 ${ }^{1}$
}

(同济大学 1. 材料科学与工程学院; 2. 先进土木工程材料教育部重点实验室, 上海 201804)

摘 要: 以壳聚糖-甘油磷酸钠-柠檬酸溶液为液相, 制备了一种新型可注射的嗍酸盐玻璃骨水泥, 通过维卡仪、万能 试验机、XRD、FTIR、SEM-EDS 等探究了液相中柠檬酸浓度 $(0.1,0.2$ 和 $0.4 \mathrm{~g} / \mathrm{mL})$ 对嗍酸盐玻璃骨水泥性能的影响。 结果表明: 柠檬酸浓度显著影响骨水泥的凝结时间和可注射性。柠檬酸浓度为 $0.2 \mathrm{~g} / \mathrm{mL}$ 时获得的骨水泥的凝结时 间最短，为 $(16 \pm 0.5) \mathrm{min}$; 可注射性最好，接近 $100 \%$ 。骨水泥压缩强度随柠檬酸浓度增大而增强，最大可达 (26.7 \pm 1.9$) \mathrm{MPa}$ 。SEM 照片显示骨水泥中生成了许多纳米微粒。XRD、FTIR 和 EDS 等结果证明, 这些纳米微粒主 要是硼酸盐、磷酸盐和柠檬酸盐等物质, 而且柠檬酸浓度能够影响骨水泥中硼酸盐晶体的形成。此外, 柠檬酸能够 加速玻璃颗粒在磷酸钠盐缓冲液中的降解速率。

关 键 词: 柠檬酸; 嗍酸盐玻璃; 骨水泥; 性能

中图分类号: TB332 文献标识码: A

\section{Effect of Citric Acid Concentration on the Properties of Borate Glass Bone Cement}

\begin{abstract}
LI Hai-Bin ${ }^{1}$, WANG De-Ping ${ }^{1,2}$, WU Ying-Ying ${ }^{1}$, YAO Ai-Hua ${ }^{1}$, YE Song ${ }^{1}$
(1. School of Materials Science and Engineering, Tongji University, Shanghai 201804, China; 2. Key Laboratory of Advanced Civil Engineering Material (Tongji University) Minisry of Education, Shanghai 201804, China)

Abstract: A novel injectable borate glass bone cement (BGBC) was prepared by using citric acid- $\beta$ glycerophosphate-chitosan solution as setting solution. The effect of citric acid $\left(\mathrm{H}_{3} \mathrm{Cit}\right)$ concentration $(0.1,0.2$ and $0.4 \mathrm{~g} / \mathrm{mL})$ on the properties of BGBC was investigated by using vicat apparatus, universal testing machine, XRD, FTIR, and SEM-EDS measurements. The experimental results indicate that $\mathrm{H}_{3} \mathrm{Cit}$ concentration significantly influences setting time and injectability of BGBC. At the $\mathrm{H}_{3}$ Cit concentration of $0.2 \mathrm{~g} / \mathrm{mL}$, BGBC obtains the shortest setting time of $(16 \pm 0.5)$ min and best injectability, which is almost fully injectable. It compressive strength increases with the increase of $\mathrm{H}_{3} \mathrm{Cit}$

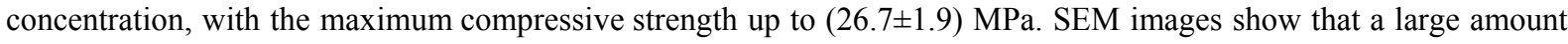
of nanoparticles are formed in the BGBC. XRD, FTIR and EDS results confirm that these nanoparticles contain borates, phosphates and phosphates. Furthermore, formation of borate crystals in the BGBC is influenced by $\mathrm{H}_{3} \mathrm{Cit}$ concentration. Immersion experiment of borate glass particles into phosphate buffer with different $\mathrm{H}_{3} \mathrm{Cit}$ concentrations shows that the degradation of borate glass is accelerated by $\mathrm{H}_{3} \mathrm{Cit}$.
\end{abstract}

Key words: citric acid; borate glass; bone cement; property

由创伤、肿瘤、感染和先天性疾病等造成的骨缺＼cjkstart损在骨科和硕面外科手术中是一种很常见的病例 ${ }^{[1]}$ 。在

收稿日期: 2016-11-01; 收到修改稿日期：2016-12-14

基金项目: 国家自然科学基金(51372170,81572105) National Natural Science Foundation of China (51372170,81572105)

作者简介: 李海滨(1991-), 男, 硕士研究生. E-mail: 1hb9@sina.cn

通讯作者: 王德平, 教授. E-mail: wdpshk@tongji.edu.cn 
骨缺损的治疗中，骨水泥由于具有可微创注射，能够 填充不规则形状和尺寸的骨缺损，且可以形成良好的 生物材料一骨组织结合等优点而被广泛研究和应用 ${ }^{[2]}$ 。 但是，临床上被广泛应用的 PMMA、硫酸钻和磷酸钙 等骨水泥依然存在各自的缺点, 例如, PMMA 骨水泥 在固化时会放出大量热且难以生物降解; 硫酸钻骨水 泥缺乏生物活性和机械强度低 ${ }^{[3]}$; 磷酸钙骨水泥降解 太慢等 ${ }^{[4]}$ 。这些缺点限制了它们的应用。

近年来, 崔旭等 ${ }^{[5-6]}$ 选用具有良好骨传导和骨 诱导性能的嗍酸盐玻璃作为固相, 制备了一种新型 的玻璃基骨水泥。该骨水泥具有良好的可注射性、 机械强度和降解性能, 固化过程中放热十分温和, 拥有良好的药物缓释性能 ${ }^{[7]}$, 且含锶嗍酸盐玻璃骨 水泥在兔股骨髁缺陷模型实验中表现出良好的成骨 性能 ${ }^{[8]}$ 。这些研究表明硓酸盐玻璃骨水泥具有较好 的应用前景。为了满足临床应用对骨水泥凝结时间、 可注射性和强度等性质的综合要求, 还需要进一步 完善骨水泥的制备工艺。而在探究实验中, 我们发 现柠檬酸浓度对嗍酸盐玻璃骨水泥性能的影响甚 大。因此, 本工作拟就此展开比较详细的探究, 并对 其原因进行分析。

\section{1 实验方法}

\section{1 试剂}

碳 酸钠 $\left(\mathrm{Na}_{2} \mathrm{CO}_{3}\right)$, 碳酸钾 $\left(\mathrm{K}_{2} \mathrm{CO}_{3}\right)$, 碳酸钙 $\left(\mathrm{CaCO}_{3}\right)$, 碳酸锶 $\left(\mathrm{SrCO}_{3}\right)$, 碱式碳酸镁 $\left(\left(\mathrm{MgCO}_{3}\right)_{4} \cdot \mathrm{Mg}(\mathrm{OH})_{2} \cdot 5 \mathrm{H}_{2} \mathrm{O}\right)$, 磷 酸 二 氢 钠 $\left(\mathrm{NaH}_{2} \mathrm{PO}_{4} \cdot 2 \mathrm{H}_{2} \mathrm{O}\right)$, 碀酸 $\left(\mathrm{H}_{3} \mathrm{BO}_{3}\right)$, 磷酸氢二钠 $\left(\mathrm{Na}_{2} \mathrm{HPO}_{4}\right)$, 柠檬酸 $\left(\mathrm{C}_{6} \mathrm{H}_{8} \mathrm{O}_{7} \cdot \mathrm{H}_{2} \mathrm{O}\right), \beta$-甘油磷酸钠等 分析纯物质和壳聚糖(BR)均购于上海国药集团化学 试剂有限公司。

\section{2 骨水泥的制备}

采用高温熔融冷却法, 在 $1100^{\circ} \mathrm{C}$ 熔制 $2 \mathrm{~h}$ 制备 $6 \mathrm{Na}_{2} \mathrm{O} \cdot 8 \mathrm{~K}_{2} \mathrm{O} \cdot 8 \mathrm{MgO} \cdot 16 \mathrm{CaO} \cdot 6 \mathrm{SrO} \cdot 54 \mathrm{~B}_{2} \mathrm{O}_{3} \cdot 2 \mathrm{P}_{2} \mathrm{O}_{5}(\mathrm{~mol} \%)$ 组成的嗍酸盐玻璃(BG), 破碎、过篮后得到的 $\mathrm{BG}$ 颗粒 $(d \leqslant 30 \mu \mathrm{m})$ 作为骨水泥的固相。将一定量的柠 檬酸 $\left(\mathrm{H}_{3} \mathrm{Cit}\right) 、 \beta$-甘油磷酸钠 $(\mathrm{GP})$ 和壳聚糖 $(\mathrm{CS})$ 依次 加入到 $10 \mathrm{~mL}$ 去离子水中, 磁力搅拌 $1 \mathrm{~h}$ 得到均匀 溶液作为骨水泥的液相。将 $1 \mathrm{~mL}$ 液相与 $2 \mathrm{~g} \mathrm{BG}$ 粉 末置于玛瑙研钵中混合, 搅拌 $60 \mathrm{~s}$ 后, 将骨水泥浆 体转移到 $6 \mathrm{~mm} \times 6 \mathrm{~mm} \times 12 \mathrm{~mm}$ 的聚四氟乙烯圆柱 形模具中, 压实抹平, 在 $37^{\circ} \mathrm{C}$ 恒温恒湿箱中固化 $1 \mathrm{~d}$, 得到骨水泥样品。改变液相中 $\mathrm{H}_{3} \mathrm{Cit}$ 的浓度, 可以得 到不同液相组成的骨水泥样品, 如表 1 所示。
表 1 样品的液相组成

Table 1 Liquid composition of samples

\begin{tabular}{cccc}
\hline Sample & $\mathrm{H}_{3} \mathrm{Cit} /\left(\mathrm{g} \cdot \mathrm{mL}^{-1}\right)$ & $\mathrm{CS} /\left(\mathrm{g} \cdot \mathrm{mL}^{-1}\right)$ & $\mathrm{GP} /\left(\mathrm{g} \cdot \mathrm{mL}^{-1}\right)$ \\
\hline $\mathrm{S} 1$ & 0.1 & 0.005 & 0.056 \\
$\mathrm{~S} 2$ & 0.2 & 0.005 & 0.056 \\
$\mathrm{~S} 3$ & 0.4 & 0.005 & 0.056 \\
\hline
\end{tabular}

\section{3 骨水泥的性能表征}

骨水泥的凝结时间用维卡仪(质量 $300 \mathrm{~g}$, 针头 直径 $1.13 \mathrm{~mm}$ )测定。以液相加入到固相的时刻为起 点, 当针头不能在骨水泥样品表面留下明显痕迹时 (压痕 $\leqslant 0.5 \mathrm{~mm}$ ) 可认为骨水泥已经凝结。

骨水泥的可注射性测试: 将 $3 \mathrm{~g}$ 新制骨水泥浆 体搅拌均匀后, 转移到 $5 \mathrm{~mL}$ 医用注射器(针筒内径 $12 \mathrm{~mm}$, 针头内径 $1.7 \mathrm{~mm}$ )内, 以 $15 \mathrm{~mm} / \mathrm{min}$ 的速率 注射骨水泥, 并通过万能试验机(CTM2500)记录注 射压力和位移的关系, 直至注射压力达到 $100 \mathrm{~N}^{[9]}$ 。 将注射出的骨水泥的质量百分数作为可注射性, 将 可注射时的最大压力作为注射力。

骨水泥的强度测试: 将试样两端面磨成平行面, 在万能试验机上测定, 加载速度为 $0.5 \mathrm{~mm} / \mathrm{min}$ 。每 组样品测试 4 次。

采用 X 射线衍射仪(XRD，D/max 2550, Japan) 对样品进行物相分析; 采用红外光谱仪 (FTIR, EQUINOXSS, Germany)测试样品的组成; 采用扫描 电子显微镜(FESEM-EDS, Hitachi S-4700)观察样品 的断面形貌。

\subsection{BG 在含 $\mathrm{H}_{3} \mathrm{Cit}$ 的磷酸钠盐缓冲液中的降 解测试}

为了探究 $\mathrm{H}_{3} \mathrm{Cit}$ 浓度对 $\mathrm{BG}$ 颗粒降解快慢的影 响, 首先配置 $1000 \mathrm{~mL} 0.2 \mathrm{~mol} / \mathrm{L}, \mathrm{pH}$ 为 7.4 的 $\mathrm{Na}_{2} \mathrm{HPO}_{4}-\mathrm{NaH}_{2} \mathrm{PO}_{4}$ 缓冲液, 再将一定量的 $\mathrm{H}_{3} \mathrm{Cit}$ 溶 解到缓冲液中, 分别配置 $\mathrm{H}_{3} \mathrm{Cit}$ 浓度为 $0 、 0.1 、 0.2$ 和 $0.4 \mathrm{~g} / \mathrm{mL}$ 的缓冲液(分别记为 PBS-0、PBS-0.1、 PBS-0.2、PBS-0.4)。将 $0.5 \mathrm{~g}$ 粒径在 $200 \sim 300 \mu \mathrm{m}$ 的 $\mathrm{BG}$ 颗粒分别浸没在 $10 \mathrm{~mL}$ 上述溶液中, 在特定时 间用 $\mathrm{pH}$ 计和电感耦合等离子发射光谱仪测定浸泡 液的 $\mathrm{pH}$ 以及溶液中 $\mathrm{B}^{3+}$ 浓度, 并将剩余的 $\mathrm{BG}$ 颗粒 取出干燥，并测定其质量。

\section{2 结果与讨论}

\section{1 $\mathrm{H}_{3} \mathrm{Cit}$ 浓度对凝结时间、可注射性和强度 的影响}

表 2 给出了样品的凝结时间和可注射性。随着 $\mathrm{H}_{3} \mathrm{Cit}$ 浓度增加, 骨水泥的凝结时间先快速缩短, 再 
略微增加。当 $\mathrm{H}_{3} \mathrm{Cit}$ 浓度为 $0.2 \mathrm{~g} / \mathrm{mL}$ 时 $(\mathrm{S} 2)$, 凝结时 间最短, 为 $(16 \pm 0.5) \mathrm{min}$ 。图 1 给出了注射不同样品 时注射压力和位移的关系曲线。结合表 1 可知: S1 的注射力最大, 为 $86.5 \mathrm{~N}$, 可注射性为 $(75 \pm 7) \% ; \mathrm{S} 2$ 注射性能最好, 注射力为 $17.6 \mathrm{~N}$, 可注射性接近 $100 \%$; S3 注射性最差，仅有 $35 \%$ 左右的骨水泥被注 射出来。在实验过程中, $\mathrm{S} 1$ 在 $\mathrm{A}$ 点出现固液分离现 象, 这是水泥浆体固体颗粒和液相粘结力较小的缘 故; $\mathrm{S} 2$ 在 $\mathrm{B}$ 点基本全部被注射出来; $\mathrm{S} 3$ 在 $\mathrm{C}$ 点难以 注射, 主要是因为浆体粘度较大, 出现成团现象。

骨水泥固化 $1 \mathrm{~d}$ 后的压缩强度如图 2 所示。 $\mathrm{H}_{3} \mathrm{Cit}$ 浓度的增加显著提高了骨水泥的抗压强度, 最大压 缩强度可以达到(26.7 \pm 1.9$) \mathrm{MPa}$ 。

表 2 样品的凝结时间和可注射性

Table 2 Setting time and injectability of samples

\begin{tabular}{cccc}
\hline Sample & Setting time/min & Injectability/\% & Injection force/N \\
\hline S1 & $40 \pm 2.0$ & $75 \pm 7$ & 86.5 \\
S2 & $16 \pm 0.5$ & $>95$ & 28.1 \\
S3 & $20 \pm 1.0$ & $35 \pm 10$ & 17.6 \\
\hline
\end{tabular}

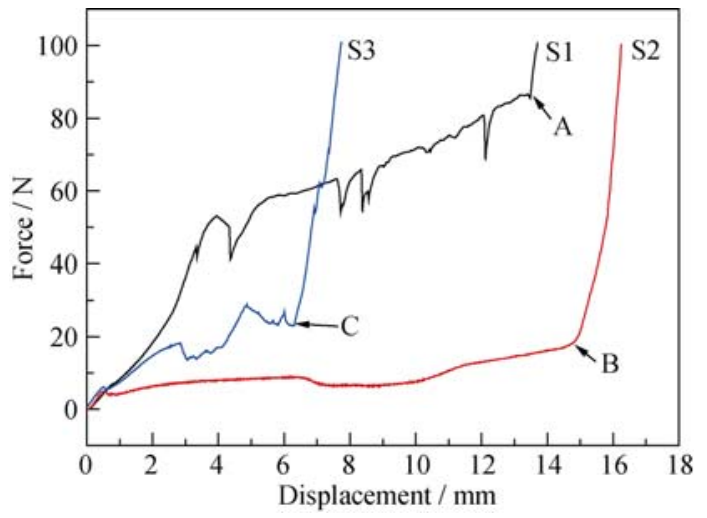

图 1 样品的注射曲线

Fig. 1 Injection curve of samples

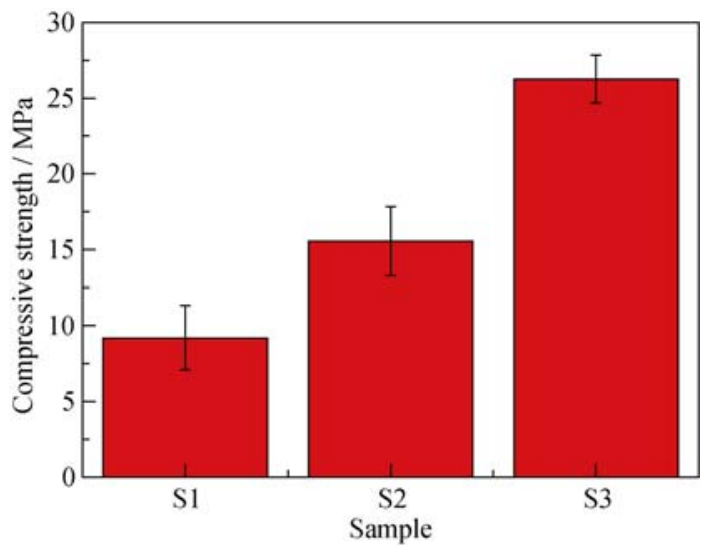

图 2 样品的压缩强度

Fig. 2 Compressive strength of samples

\section{2 骨水泥固化产物的物相分析}

样品的 XRD 图谱如图 3 所示, 与 $\mathrm{BG}$ 的 XRD 图谱相比, $\mathrm{S} 1$ 的图谱出现了 $\mathrm{Na}_{2} \mathrm{~B}_{4} \mathrm{O}_{5}(\mathrm{OH})_{4} \cdot 8 \mathrm{H}_{2} \mathrm{O}$ 的 特征峰, 表明固化产物中有 $\mathrm{Na}_{2} \mathrm{~B}_{4} \mathrm{O}_{5}(\mathrm{OH})_{4} \cdot 8 \mathrm{H}_{2} \mathrm{O}$ 晶 体生成。但随着柠檬酸浓度的增加, S2 的图谱中 $\mathrm{Na}_{2} \mathrm{~B}_{4} \mathrm{O}_{5}(\mathrm{OH})_{4} \cdot 8 \mathrm{H}_{2} \mathrm{O}$ 的衍射峰明显减弱; 而 $\mathrm{S} 3$ 图谱 中几乎没有明显的晶体衍射峰。这与 $\mathrm{H}_{3} \mathrm{Cit}$ 浓度的 提高增强了液相酸性, 在一定程度上抑制了 $\mathrm{Na}_{2} \mathrm{~B}_{4} \mathrm{O}_{5}(\mathrm{OH})_{4} \cdot 8 \mathrm{H}_{2} \mathrm{O}$ 晶体的生成有关。

图 4 为样品的红外图谱, 从图中可以看到, $\mathrm{BG}$ 的红外吸收图谱中主要有 $\left[\mathrm{BO}_{3}\right]$ 中 $\mathrm{B}-\mathrm{O}$ 的伸缩振动 峰 $\left(1383 \mathrm{~cm}^{-1}\right)$ 和弯曲振动峰 $\left(716 \mathrm{~cm}^{-1}\right)$, 以及 $\left[\mathrm{BO}_{4}\right]$ 中 $\mathrm{B}-\mathrm{O}$ 的振动吸收 $\left(997 \mathrm{~cm}^{-1}\right)^{[10]}$ 。与 $\mathrm{BG}$ 相比, 骨水 泥样品的红外图谱中, 716 和 $1383 \mathrm{~cm}^{-1}$ 处的峰分裂 成了两个峰 $\left(696\right.$ 和 $730 \mathrm{~cm}^{-1}, 1353$ 和 $\left.1386 \mathrm{~cm}^{-1}\right)$ 。这 是因为骨水泥中生成了硼酸盐, 而硼酸盐中存在的 氢键会导致嗍氧基团中 $\mathrm{B}-\mathrm{O}$ 键发生能级分裂，从而 使嗍氧基团产生不同的吸收 ${ }^{[11]}$ 。560 和 $1022 \mathrm{~cm}^{-1}$ 处 的峰是 $\mathrm{PO}_{4}{ }^{3-}$ 的振动峰 ${ }^{[10]}$ 。结合 $\mathrm{BG}$ 的红外图谱可知,

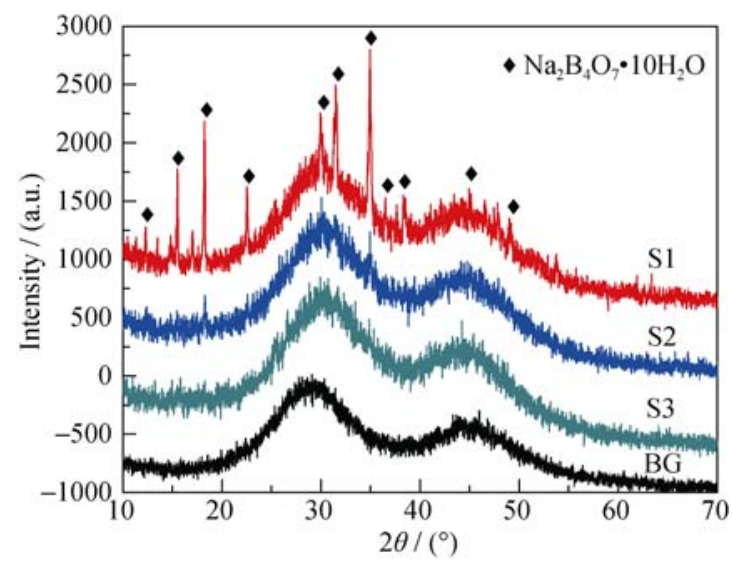

图 3 样品的 XRD 图谱

Fig. 3 XRD patterns of samples

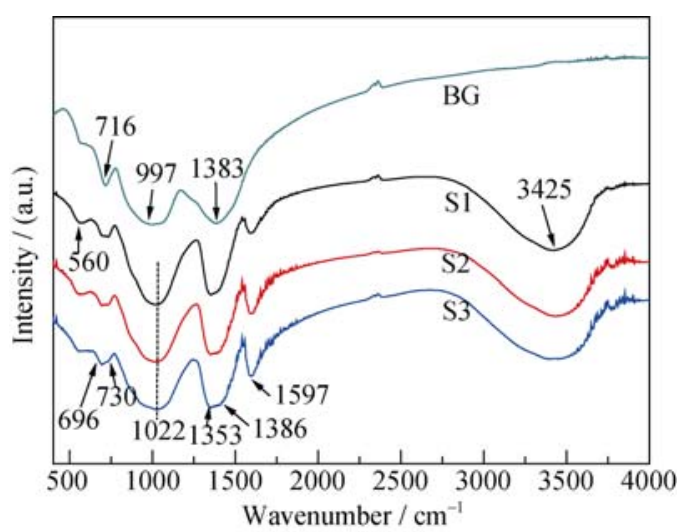

图 4 样品的红外图谱

Fig. 4 Infrared spectra of samples 
$1022 \mathrm{~cm}^{-1}$ 宽且强的峰也是 $\mathrm{PO}_{4}{ }^{3-}$ 和 $\mathrm{BO}_{4}{ }^{5-}$ 基团的重叠 峰。骨水泥样品中也出现了 $-\mathrm{COO}^{-}$和 $\mathrm{H}_{2} \mathrm{O}$ 的振动峰 (依次在 1597 和 $3425 \mathrm{~cm}^{-1}$ 左右), $-\mathrm{COO}^{-}$的振动峰随 $\mathrm{H}_{3} \mathrm{Cit}$ 浓度的增加而增强。红外图谱表明骨水泥中存 在硼酸盐、柠檬酸盐、磷酸盐等多种成分的物质。

\section{3 骨水泥断面形貌观察}

骨水泥固化 $1 \mathrm{~d}$ 后将其折断, 干燥喷金后用 SEM 观察其断面形貌, 结果如图 5 所示。从图 5 中 可以看到, 部分反应的 $\mathrm{BG}$ 颗粒分散在一个连续相 中连续相中含有大量的纳米微粒。 $\mathrm{BG}$ 颗粒和这些 纳米微粒之间存在一些裂缝, 这是在样品干燥过程 中毛细管力所导致的 ${ }^{[5]}$ 。由 EDS 结果可知, 纳米微 粒由大量的 $\mathrm{B}$ 和 $\mathrm{O}$ 以及少量 $\mathrm{Na} 、 \mathrm{~K} 、 \mathrm{Ca} 、 \mathrm{Sr} 、 \mathrm{Mg}$ 、 $\mathrm{C}$ 和 $\mathrm{P}$ 元素组成。结合 XRD 和 FTIR 的结果可知, 这 些纳米微粒是从 $\mathrm{BG}$ 释放出的金属离子和 $\mathrm{B}^{3+}$

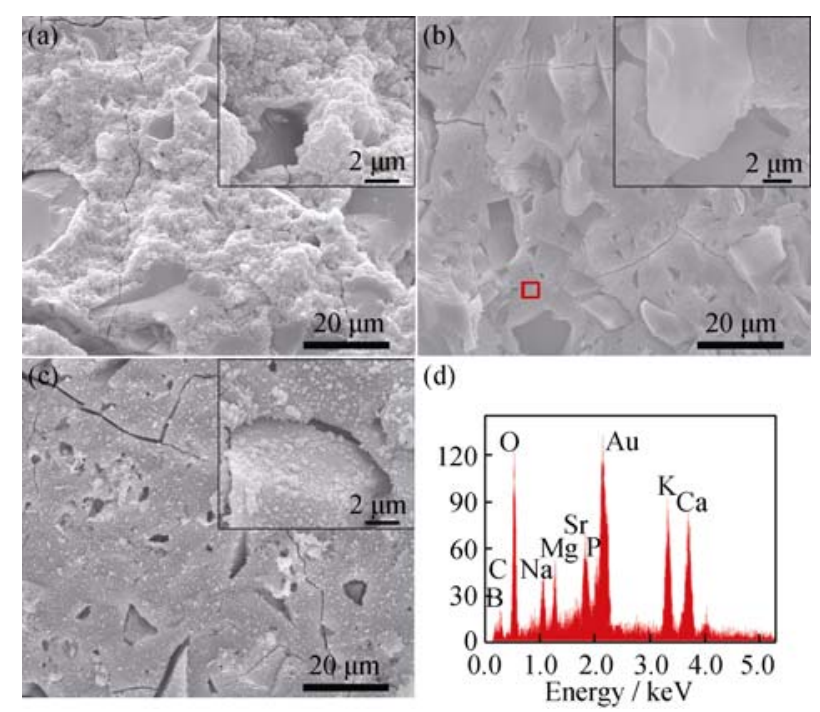

图 5 样品断面的 SEM 照片((a) S1; (b) S2; (c) S3)和图(b)中红 色方框区域的能谱图

Fig. 5 SEM images of the fracture surfaces of samples ((a) S1; (b) S2; (c) S3) and EDS pattern of the boxed area in Fig. (b)

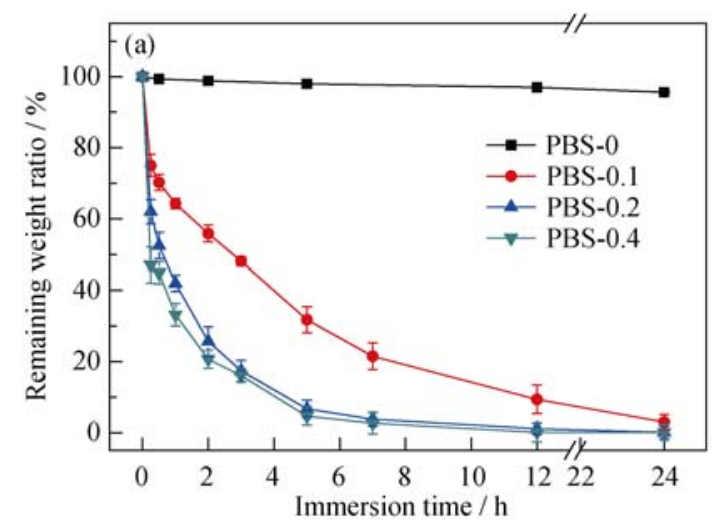

与液相中的 $\mathrm{PO}_{4}{ }^{3-}$ 和柠檬酸根等离子结合的产物, 它们主要是硼酸盐和少部分柠檬酸盐以及磷酸盐物 质。可以发现, S3 中生成的纳米微粒明显多于 $\mathrm{S} 1$ 和 $\mathrm{S} 2$, 在 BG 表面仍紧密结合了许多纳米微粒。这部 分纳米微粒是 $\mathrm{BG}$ 表面的离子与溶液中离子反应的 产物, 它们增强了 $\mathrm{BG}$ 颗粒与连续相的结合力。连续 相中，纳米微粒也随着 $\mathrm{H}_{3} \mathrm{Cit}$ 浓度的增大而结合得 更紧密。

\section{$2.4 \mathrm{BG}$ 在含 $\mathrm{H}_{3} \mathrm{Cit}$ 的磷酸盐缓冲液中的降解} 性能分析

BG 颗粒在 PBS-0、PBS-0.1、PBS- 0.2 和 PBS- -0.4 溶液中的降解曲线以及浸泡液中 $\mathrm{B}$ 离子浓度变化情 况如图 6 所示。BG 在 PBS-0 溶液中缓慢降解，降解 $1 \mathrm{~d}$ 后, 质量还剩余 $95.6 \%$; 而 $\mathrm{H}_{3} \mathrm{Cit}$ 的添加能够急 剧加速 $\mathrm{BG}$ 的降解速度, 特别是在 PBS-0.2 和 PBS-0.4 中浸没 $7 \mathrm{~h}, \mathrm{BG}$ 就基本完全降解了。而浸泡 液中 $\mathrm{B}$ 离子的浓度也快速增加。与此同时，随着 $\mathrm{BG}$ 的降解, 浸泡液的 $\mathrm{pH}$ 逐渐增大, 如图 7 所示。其中 PBS- 0.4 溶液因酸性过大，导致 $\mathrm{pH}$ 变化相对较小。 浸泡液 $\mathrm{pH}$ 上升是因为 $\mathrm{BG}$ 中金属离子和 $\mathrm{B}$ 离子的 快速溶出, 导致溶液中 $\mathrm{H}^{+}$浓度减少所致 ${ }^{[5]}$ 。

\section{5 凝结机理分析}

图 8 显示了 $\mathrm{BG}$ 固相在 CS-GP-H ${ }_{3}$ Cit 溶液中的 凝结过程。如图 8(a)所示, 由于 BG 的结构不稳定, 在溶液中金属离子(如 $\mathrm{Ka}^{+} 、 \mathrm{Na}^{+} 、 \mathrm{Ca}^{2+}$ 等)和 $\mathrm{B}^{3+}$ 会从 $\mathrm{BG}$ 表面释放出来，导致溶液中 $\mathrm{pH}$ 的升高。与此同 时, $\mathrm{Ca}^{2+}$ 将与溶液中的 $\mathrm{PO}_{4}{ }^{3-}$ 和 $\mathrm{OH}^{-}$等离子作用生成 磷酸盐物质 ${ }^{[12]}$ 。此外, 溶液中 $\mathrm{CS}$ 的粘度也会随着 溶液 $\mathrm{pH}$ 逐渐增大而增加, 并最终发生溶胶-凝胶转 变, 促进骨水泥的凝结 ${ }^{[5]}$ 。

如图 8(b)所示, 在溶液中增加 $\mathrm{H}_{3} \mathrm{Cit}$ 的浓度, 一 方面增加了溶液的酸性, 加速 $\mathrm{BG}$ 中离子的溶出;

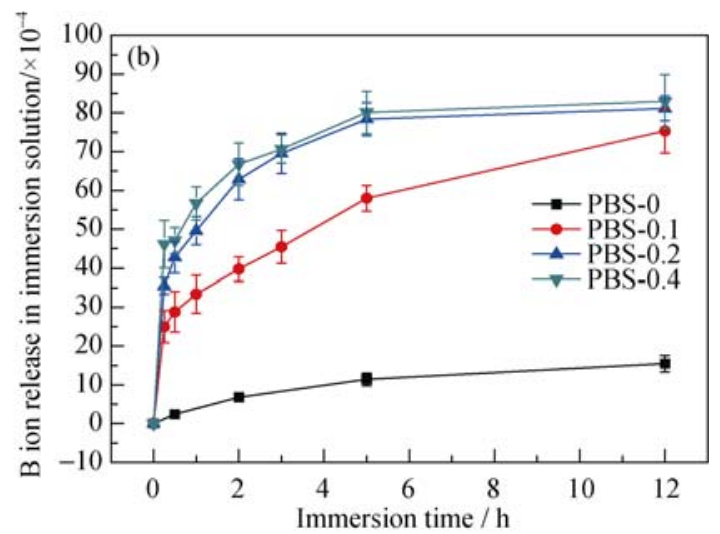

图 6 嗍酸盐玻璃在 PBS-0、PBS-0.1、PBS-0.2 和 PBS-0.4 溶液中的降解曲线(a)以及浸泡液中 B 离子释放浓度(b)

Fig. 6 Degradation curves of borate glass in the PBS-0, PBS-0.1, PBS-0.2 and PBS-0.4 (a) and the B ion release concentration in the immersion solution (b) 


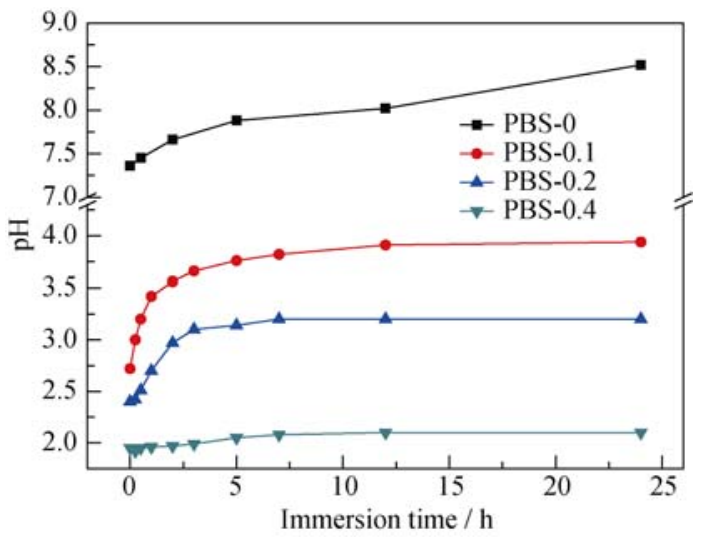

图 7 浸泡液的 $\mathrm{pH}$ 随浸泡时间的变化情况

Fig. 7 Variation of $\mathrm{pH}$ of immersion solutions with the immersion time

且柠檬酸根离子具有螯合作用, 也会与 $\mathrm{Ca}^{2+} 、 \mathrm{Mg}^{2+}$ 等 金属离子结合 ${ }^{[9]}$, 生成柠檬酸盐物质。而这种螯合作 用, 又降低了液相中这些金属离子的有效浓度, 从而 进一步加速了 $\mathrm{BG}$ 中离子的释放。释放出来的离子最 终生成嗍酸盐、磷酸盐和柠檬酸盐等纳米微粒, 增加 了骨水泥浆体的粘度, 与 CS 共同形成了骨水泥中的 连续相。故随着 $\mathrm{H}_{3} \mathrm{Cit}$ 浓度的增加, $\mathrm{BG}$ 中释放出的离 子数目增多, 骨水泥中最终生成的纳米微粒也相应增 加, 这就促进了骨水泥的凝结, 增强了连续相的强度, 增大了连续相与 $\mathrm{BG}$ 颗粒的结合力。这些原因导致 $\mathrm{S} 3$ 和 $\mathrm{S} 2$ 比 $\mathrm{S} 1$ 凝结时间明显缩短, $\mathrm{S} 1 、 \mathrm{~S} 2$ 和 $\mathrm{S} 3$ 强度依 次增加。 $\mathrm{S} 2$ 则因为骨水泥浆体粘度以及 $\mathrm{BG}$ 颗粒和液 相结合力大小最佳, 拥有最好的可注射性。

另一方面, $\mathrm{H}_{3} \mathrm{Cit}$ 浓度的增加导致溶液酸性增 强, 增大了 CS 的溶解度, 降低了液相粘度, 延缓了 $\mathrm{CS}$ 的溶胶-凝胶转变。并且溶液中酸性过强并不利
于嗍酸盐、磷酸盐物质的生成析出, 且柠檬酸根的 螯合作用也会影响 $\mathrm{PO}_{4}{ }^{3-}$ 与 $\mathrm{Ca}^{2+}$ 的作用。另外, 溶液 中嗍氧基团的存在形式以及嗍酸盐的结晶析出受到 溶液中嗍氧基团浓度、溶液酸碱度等条件的影响 ${ }^{[13]}$ 。 从这方面来说, $\mathrm{H}_{3} \mathrm{Cit}$ 浓度的增大在一定程度上延缓 了骨水泥的凝结, 也抑制了硼酸盐的结晶和磷酸盐 的析出。这也是 S3 比 S2 凝结时间略微增加的原因, 同时解释了随着 $\mathrm{H}_{3}$ Cit 浓度增加, $\mathrm{S} 2$ 和 $\mathrm{S} 3$ 的 XRD 图谱中 $\mathrm{Na}_{2} \mathrm{~B}_{4} \mathrm{O}_{5}(\mathrm{OH})_{4} \cdot 8 \mathrm{H}_{2} \mathrm{O}$ 的衍射峰逐渐减小乃至 消失的现象。

图 8(c)显示导致硼酸盐骨水泥凝结的主要作用: $\mathrm{BG}$ 中溶出离子与溶液中各种离子的反应、 $\mathrm{CS}$ 的溶 胶-凝胶转变以及部分反应的 $\mathrm{BG}$ 颗粒、 $\mathrm{CS}$ 与生成 的纳米微粒间的相互作用。图 8(d)则是骨水泥凝结 状态示意图。

\section{3 结论}

以 CS-GP- $\mathrm{H}_{3}$ Cit 溶液为液相，制备了可注射的 嗍酸盐玻璃骨水泥。液相中 $\mathrm{H}_{3} \mathrm{Cit}$ 浓度对骨水泥的 性能影响显著。 $\mathrm{H}_{3} \mathrm{Cit}$ 浓度的增加能够加快 $\mathrm{BG}$ 中金 属离子和 $\mathrm{B}^{3+}$ 的溶出, 促进骨水泥固相和液相的反 应，提高骨水泥的强度，同时也增强了液相的酸性。 这在一定程度上影响骨水泥凝结过程中硼酸盐晶体 和磷酸盐物质的生成析出, 并延缓了 CS 的溶胶-凝 胶转变。这些因素的协同作用也导致了 $\mathrm{H}_{3} \mathrm{Cit}$ 对骨水 泥凝结时间和可注射性等性能影响的复杂性。在制 备的三个样品中, 柠檬酸浓度为 $0.2 \mathrm{~g} / \mathrm{mL}$ 时得到的 骨水泥具备最佳的综合性能，拥有较好的应用前景。

(a) The ions release from the BG

(b) $\mathrm{H}_{3}$ Cit accelerates the ions release

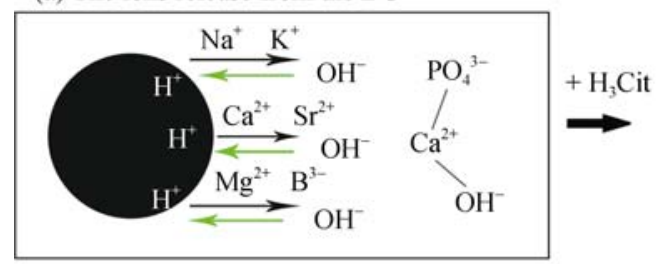

(d) Setting

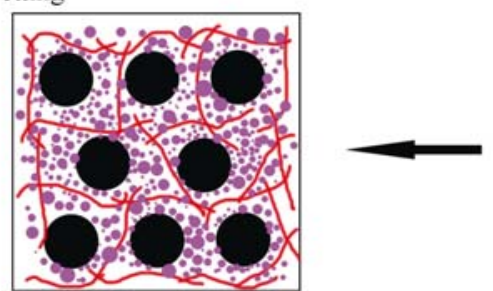

BG Generated nanoparticles

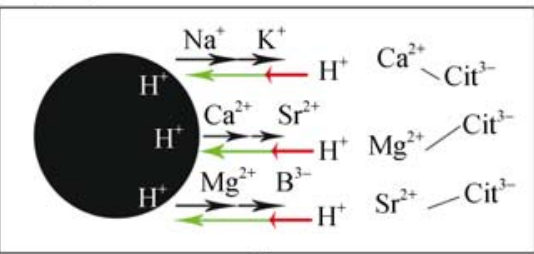

(c) Reactions in the setting process

图 8 骨水泥凝结机理示意图

Fig. 8 Schematic diagram of setting mechanism of bone cement 


\section{参考文献:}

[1] LAURENCIN C, KHAN Y, El-AMIN S F, Bone graft substitutes. Expert Rev. Med. Devices, 2006, 3(1): 49-57.

[2] KRETLOW J D, KLOUDA L, MIKOS A G. Injectable matrices and scaffolds for drug delivery in tissue engineering. Adv. Drug Deliv. Rev. 2007, 59(4/5): 263-273.

[3] DOADRIO J C, ARCOS D, CABANAS M V. Calcium sulphatebased cements containing cephalexin. Biomaterial, 2004, 25(13): 2629-2635.

[4] KUANG G M, YAU W P, LAM W M, et al. An effective approach by a chelate reaction in optimizing the setting process of strontium incorporated calcium phosphate bone cement. J. Biomed. Mater. Re. B, 2012, 100(3): 778-787.

[5] CUI XU, ZHANG YADONG, WANG HUI, et al. An injectable borate bioactive glass cement for bone repair: preparation, bioactivity and setting mechanism. Journal of Non-Crystalline Solids, 2016, 432: 150-157.

[6] CUI XU, ZHAO CUNJU, GU YIFEI, et al. A novel injectable borate bioactive glass cement for local delivery of vancomycin to cure osteomyelitis and regenerate bone. J. Mater. Sci.: Mater. Med., 2014, 25(3): 733-745.
[7] DING HAO, ZHAO CUN JU, CUI XU, et al. A novel injectable borate bioactive glass cement as an antibiotic delivery vehicle for treating osteomyelitis. Plos One, 2014, 9(1): e85472.

[8] ZHANG Y, CUI X, ZHAO S, et al. Evaluation of injectable strontium-containing borate bioactive glass cement with enhanced osteogenic capacity in a critical-sized rabbit femoral condyle defect model. ACS Appl. Mater. Interfaces, 2015, 7(4): 2393-2403.

[9] HESARAKI SAEED, ZAMANIAN ALI, MOZTARZADEH FATOLLAH. The influence of the acidic component of the gasfoaming porogen used in preparing an injectable porous calcium phosphate cement on its properties: acetic acid versus citric acid. $J$. Biomed. Mater. Res. B, 2008, 86B(1): 208-216.

[10] LIU X, RAHAMAN M N, DAY D E. Conversion of melt-derived microfibrous borate (13-93B3) and silicate (45S5) bioactive glass in a simulated body fluid. J. Mater. Sci: Mater. Med., 2013, 24(3): 583-595.

[11] 赵 翠, 任丽莉, 王 栋, 等. 煅嗍砂的红外光谱分析与评价. 光 谱学与光谱分析, 2011, 31(8): 2109-2113.

[12] HUANG W, RAHAMAN M N, DAY D E, et al. Mechanisms for converting bioactive silicate, borate, and borosilicate glasses to hydroxyapatite in dilute phosphate solutions. Phys. Chem. Glasses B, 2006, 47(6): 647-658.

[13] 戈海文, 邓天龙, 姚 燕, 等. 嗍酸盐晶体及其溶液结构研究现 状. 广东微量元素科学, 2011, 18(1): 17-23. 